\title{
Simulation of dislocation patterns in multislip
}

\author{
R. Madec, B. Devincre, L.P. Kubin * \\ Laboratoire d'Etude des Microstructures, CNRS-ONERA (OM), 29 Av. de la Division Leclerc, BP 72, 92322 Châtillon Cedex, France
}

Accepted 12 April 2002

\begin{abstract}
Dislocation dynamics simulations of multiple slip in f.c.c. crystals lead to the formation of patterned microstructures. The mechanisms participating to dislocation storage and dynamic recovery are investigated and discussed. Cross-slip and short-range interactions are found to govern the bifurcation from uniform to ordered microstructures.

(C) 2002 Acta Materialia Inc. Published by Elsevier Science Ltd. All rights reserved.
\end{abstract}

Keywords: Dislocations; Cell structures; Cross-slip; Junctions; Dynamic recovery

\section{Introduction}

Numerical simulations can provide an original insight into the mechanisms of dislocation cell formation in f.c.c. crystals. Dislocation dynamics (DD) simulations are at present limited to small strains, $<1 \%$, and small model volumes. Thus, this approach does not permit to check the occurrence of similitude or self-similarity properties in the microstructures. It allows, nevertheless, to examine the respective contributions from various dislocation processes to the emergence of dislocation patterns without making strong assumptions.

From early transmission electron microscopy studies, it is known that cell structures form at the onset of stage III of the deformation of single crystals, or at small strains in multislip conditions $[1,2]$. These last conditions are, therefore, the most suited for DD simulations. Three major contribu-

\footnotetext{
${ }^{*}$ Corresponding author.

E-mail address: kubin@onera.fr (L.P. Kubin).
}

tions to patterning have been discussed in the literature and are examined in the present work. As emphasized by Kocks [3], the storage of immobile dislocations is due to strong interactions of the mobile dislocations with a "forest" of intersecting dislocations. The cross-slip mechanism has often (but not always) been assumed to be responsible for the temperature dependence of $\tau_{\text {III }}$, the critical stress for the onset of stage III, i.e., for dynamic recovery. Within this view, the relaxation of internal stresses that characterizes stage III behavior is attributed to the mutual annihilation of mobile screw dislocations with immobile ones. In contrast, continuum spatio-temporal models tend to propose general explanations for the formation of dislocation cells. In the deterministic model by Kratochvíl and Orlová [4], an internal bending type of instability is invoked, whereas a more recent statistical model points out at long-range stress fluctuations as a possible cause of patterning, by analogy with noise-induced phase transitions [5].

In what follows, the numerical method is briefly recalled and answers provided by DD simulations 
to the three points mentioned above are presented and discussed.

\section{Dislocation dynamics simulations}

The present results were obtained with the help of DD simulations, in which the character of the dislocation lines is discretized into a succession of screw and edge segments (the "Edge-Screw" model), or of screw, mixed and edge segments (the "Mixed" model). Due to the reduction in the number of degrees of freedom resulting from the discretization of characters, this type of simulation provides an excellent compromise between numerical accuracy and computing efficiency. This makes it possible to treat dense configurations in material volumes with dimensions larger than those of a typical dislocation cell. The basic principles of these three-dimensional (3-D) simulations can be found in several references [6-9] and will not be detailed here.

As far as elastic properties are concerned, it is sufficient to mention that all the contributions to the effective stress on a dislocation segment are computed within isotropic elasticity theory. This includes a line tension stress, estimated from Foreman's formula [10], the elastic interactions with other segments from the same line or from other lines, a small Peierls stress $\left(10^{-5} \mu\right.$, where $\mu$ is the shear modulus) and the resolved applied stress. The mobility of a segment is then governed in f.c.c. crystals by a viscous drag due to the interaction with phonons and electrons.

The formation and destruction of junctions is treated as a purely elastic problem, on the base that it is governed by a balance of line energies, to which the contribution of core energies is negligible. The only assumption made is that dislocations are not dissociated, which certainly applies to an f.c.c. crystal with a high stacking fault energy (SFE) like aluminum. Several arguments show that this simplifying assumption also applies to crystals of lower SFE like copper, or even silver, as far as junction properties are concerned. For instance, a detailed comparison of the critical stress for the unzipping of Lomer-Cottrell locks shows that very similar values are found by atomistic and
DD simulations and by DD simulations of the corresponding non-dissociated configuration, the Lomer lock [11,12]. The fact that the junction strength is not sensitive to the core structure is also considered as an explanation for the relative insensitivity of stage II hardening in f.c.c. crystals to the value of the SFE.

In the present simulations, the only mechanism by which dislocations can move out of their slip plane is the cross-slip of screw dislocations. Crossslip is implemented in a stochastic manner via an Arrhenius-like probability law, which accounts for its thermally activated character. A simplified form for the activation energy is derived from the Friedel-Escaig mechanism (see e.g. [13]), in agreement with more recent atomistic simulations [14]. It depends on the local effective stresses on the slip and cross-slip planes and on the SFE of the material through the value of $\tau_{\text {III }}$. The way this local rule is numerically implemented is the same in all versions of the DD simulations $[6,9,15]$.

The initial microstructure is made up of an initially random distribution of source segments, to which a prestrain and a further relaxation are sometimes applied to simulate annealed microstructures. The model crystal is deformed with a constant imposed strain rate, typically $20 \mathrm{~s}^{-1}$, along a [0 01 1] stress axis (specific details will be given below for each set of numerical experiments). The boundary conditions are either those of free surfaces (Edge-Screw model) or periodic boundary conditions $[9,16]$ for the Mixed model. In what follows, the temperature is fixed to about $400 \mathrm{~K}$, the elastic constants of the material and the cross-slip properties being those of copper. Finally, one may keep in mind that local lattice rotations are not taken into account into these DD simulations.

\section{Junctions and dislocations storage}

In a crystal deformed in multislip conditions, dislocation storage occurs through the interaction of gliding dislocations with strong forest obstacles, particularly attractive junctions. The conditions for the formation and destruction of the three types of junctions between perfect dislocations in 


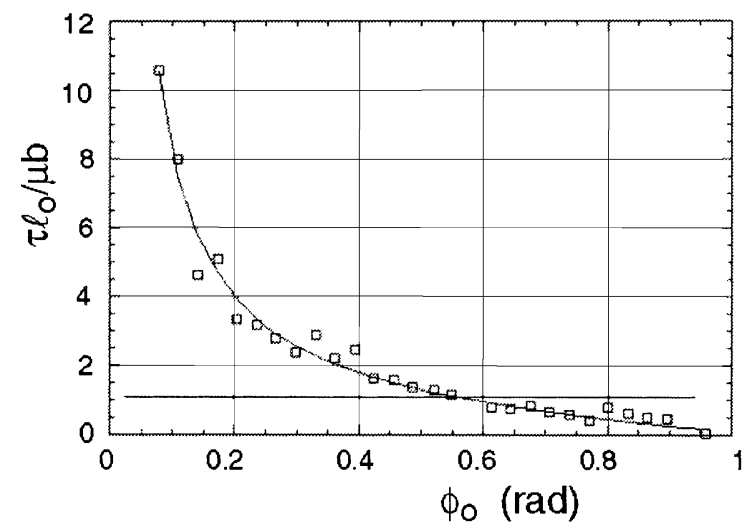

Fig. 1. The critical stress $\tau_{0}$ of a symmetrical Lomer lock, scaled by $\mu b / \ell_{0}$, where $\ell_{0}$ is the initial length of the interacting segments, as a function of the angle $\phi_{0}$ between the initially straight interacting segments and the junction direction. The squares represent simulation results obtained with the Mixed model. The curve is a fit to a simplified elastic model [9]. The horizontal line corresponds to a typical (reduced) yield stress of $2.5 \times 10^{-4} \mu$.

f.c.c. crystals (the Lomer, glissile and Hirth locks) have been investigated in detail as a function of the orientation of the interacting lines $[8,9,17]$. Fig. 1 shows a plot of the critical stress (in reduced units) for the unzipping a symmetrical Lomer lock as a function of the common initial angle $\phi_{0}$ of the interacting lines with the junction direction. The simulation results (squares) are fitted to a simplified elastic model. The latter contains one arbitrary constant but provides a surprisingly accurate prediction. One can see that there is a whole spectrum of junction strengths and a divergence when the lines are initially parallel to the junction direction $\left(\phi_{0}=0\right)$. In this particular case, the length of the unzipping arms is zero and an infinite stress has to be applied to them to destroy the junction. The horizontal line represents, in the same reduced units, the typical flow stress of an annealed f.c.c. single crystal at small strains. Thus, most junction of smaller critical stress are destroyed under stress, whereas those of larger critical stress are stored and can contribute to patterning. The flow stress is, therefore, such that a sufficient fraction of all the junctions present at a given moment can be destroyed, in order to allow the mobile density to produce the imposed plastic strain rate. Within the Mixed model, the scaling relationship $\tau=\alpha \mu b \sqrt{ } \rho_{\mathrm{f}}$ between the resolved flow stress and the square root of the forest density in multiple slip is spontaneously obtained ( $b$ is the modulus of the $1 / 2\langle 110\rangle$ Burgers vector). A mean value of $\alpha=0.38$ comes out without any adjustment [9], which compares well with the experimental data on copper and silver compiled by Basinski and Basinski [18].

\section{Influence of cross-slip on pattern formation}

Fig. 2 shows two simulated microstructures obtained in multiple slip with the Mixed model. The numerical conditions and the initial configurations are exactly the same, except that the crossslip probability is set to zero in Fig. 2a. An early stage of organization appears in Fig. 2b, where well-defined dislocation-dense regions can be observed. These regions are in the form of veins, rather than walls, with preferential $\langle 110\rangle$ directions, parallel to the intersections of the active slip systems. Their average spacing is $d \approx 10 \mu \mathrm{m}$, to be compared to the periodicity of about $10 \sqrt{2} \mu \mathrm{m}$ imposed along the $\langle 110\rangle$ directions by the boundary conditions. To fix the ideas, the axial stress is $\sigma=2.7 \times 10^{-4} \mu$ and with a Burgers vector $b=0.255 \mathrm{~nm}$, we have $d \approx 11 \mu b / \sigma$ for these emerging structures. Similar microstructures were obtained previously with the Edge-Screw model [19], in simulated volumes of $15 \mu \mathrm{m}^{3}$ with free surfaces. The dislocation-dense regions are anchored on an underlying skeleton of stable junctions; they also contain cross-slipped segments, jogs and debris. In contrast, in the absence of cross-slip, the organization is much less wellmarked and the formation of observable patterns is either delayed to larger strains or suppressed (cf. Fig. 2a). Although one may assume that the rearrangement of the microstructure by cross-slip contributes to the refinement of the dislocationdense regions and their 3-D extension, the detail of the mechanisms that cooperate to produce the pattern of Fig. 2b remain to be investigated. Thus, this numerical experiment essentially confirms that cross-slip strongly favors the emergence of ordered dislocation microstructures. Within $5 \%$, the total dislocation density is the same in the two situations 

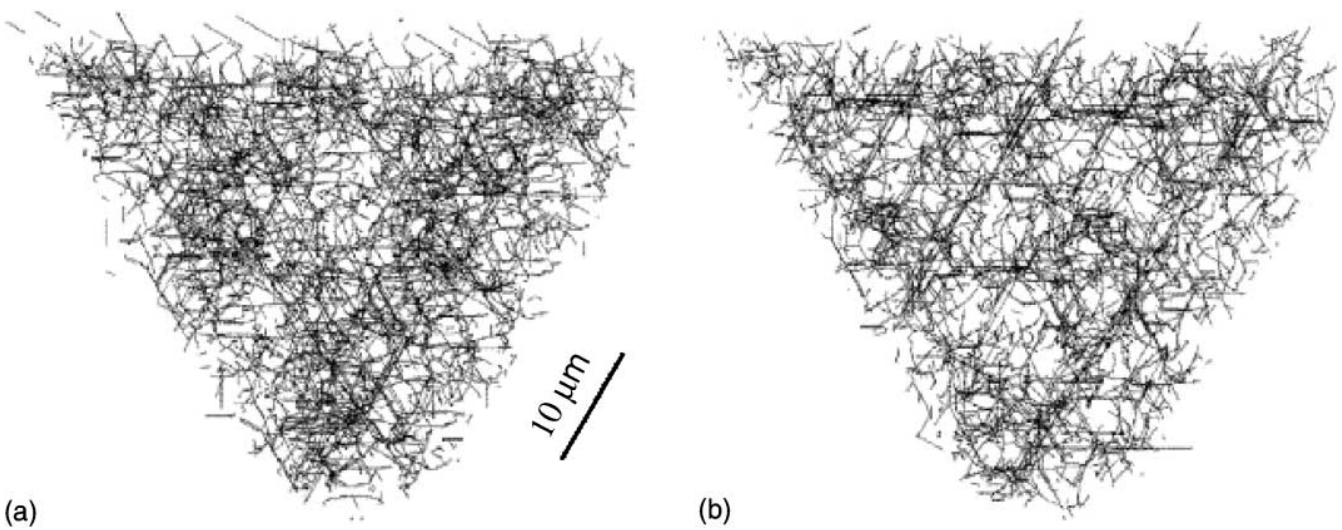

Fig. 2. (111) foils of thickness $5 \mu \mathrm{m}$ extracted at the same position from simulated crystal of approximately cubic shape $\left(9.8 \times 11.6 \times 8.4 \mu^{3}\right)$, strained along a [ 1000$]$ stress axis (the microstructures are under stress). The simulation conditions are identical (initial density $10^{12} \mathrm{~m}^{-2}$, imposed strain rate $20 \mathrm{~s}^{-1}$, total plastic strain $\varepsilon \approx 2 \times 10^{-3}$ ), except that the cross-slip probability is set to zero in (a). In (b) and in the presence of cross-slip, sharp dislocation-dense regions are observed along the three $\langle 110\rangle$ directions of the (1 11 1) plane. Sections of the simulated volume and of one layer of replicas stemming from periodic boundary conditions are shown.

depicted in Fig. 2 and the corresponding simulated stress-strain curves do not substantially differ (cf. Fig. $4 \mathrm{~b}$ below). This confirms the relative insensitivity of the flow stress on the arrangement of the microstructure, which has been noted by many authors $[20,21]$.

\section{Internal stresses}

Fig. 3a shows another emerging microstructure obtained in the presence of cross-slip, in conditions similar to those of Fig. 2b. This figure is obtained by superimposing a large number of successive gray images. The black areas are, therefore, those where storage resulted in stable dislocation-dense regions. Fig. $3 b$ shows a mapping of the resistive internal stress resolved in one of the active slip systems. The amplitude of these stresses, whose sign is opposite to that of the resolved applied stress, increases with increasing darkness up to -2 times the applied shear or more in the black areas. These negative internal stresses approximately correspond to the dislocation-poor areas of Fig. 3a. In the same way, the complementary mapping of the positive internal stresses (Fig. 3c) delineates
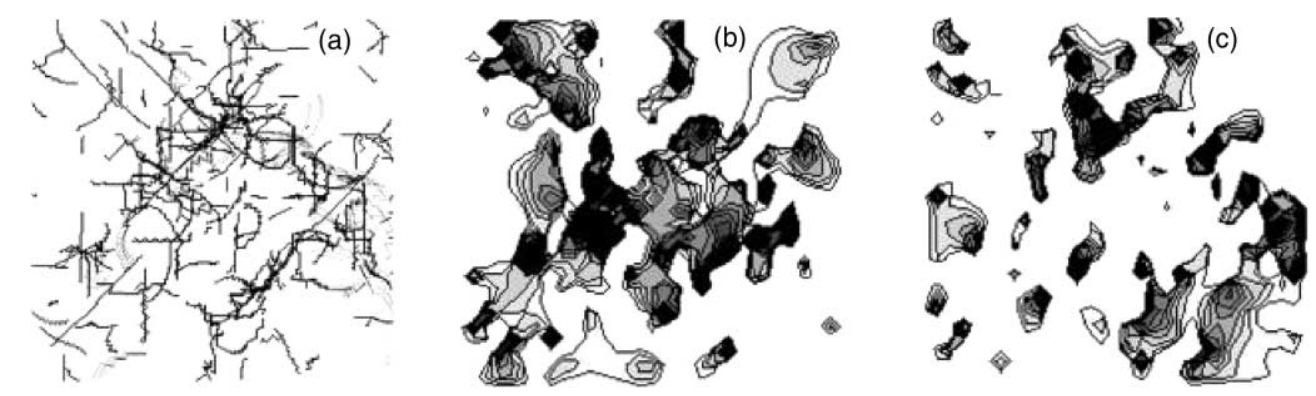

Fig. 3. (a) A simulated microstructure obtained from the Edge-Screw model in conditions similar to those of Fig. 2b ([1 00$]$ foil of thickness $2 \mu \mathrm{m}$ extracted from a simulation box of linear dimension $15 \mu \mathrm{m}$ ). This picture was obtained by superimposing successive gray images, in order to better visualize the regions where dislocations are permanently stored. The dense veins are parallel to the $\langle 110\rangle$ directions of the foil plane. (b) A mapping of the resistive (negative) internal shear stresses in one of the active slip plane (the dark areas are those where the modulus of the internal stress is the largest). (c) A mapping of the positive internal stresses on the same slip plane. 
areas that approximately correspond to the dislocation-dense regions. This picture is qualitatively consistent with Mughrabi's composite model [19], according to which the continuity of plastic strain between the hard dislocation-dense regions and the soft dislocation-poor regions induces positive internal stresses in the former and negative ones in the latter. The high local stress levels in the walls enhance the cross-slip probability, since the latter is stress-dependent. We attribute to this effect the progressive refinement of the walls, which can be seen by comparing the gray and dark contrasts in Fig. 3a, and their stabilization by the relaxation of the local peaks of internal stress.

\section{Influence of long- and short-range stresses on pattern formation}

Numerical experiments were designed in order to check the influence of close and distant interactions between dislocations on the evolution of the microstructure and on the flow stress [7]. A cutoff radius was introduced of the order of $\rho^{-1 / 2}$ (about $1 \mu \mathrm{m}$ ). In one test, only the elastic interactions situated inside this cut-off were computed and in a second one only those situated outside the cutoff. In both case intersection and junction processes were included. Fig. 4a shows a microstructure obtained in the case where short-range elastic interactions are cut out. The dislocations are uniformly distributed in space and no trace of emerging structure is found. In the case where only shortrange interactions are accounted for, a central dislocation-poor region is formed [7]. Its radius is larger than the cut-off radius, so that this structure is not an artifact due to the truncation of the elastic interactions (in contrast to a common belief, such artifacts arise only on microstructures that are let to relax in the absence of applied stress). These results are interpreted as follows. The largest internal stresses are produced by short-range dislocation interactions. Since the cross-slip probability is strongly stress-sensitive, omitting these interactions is equivalent to de-activating cross-slip. As shown above, this prevents the emergence of an organized microstructure. Thus, we conclude that, although long-range interactions do play a role once dislocation patterns are well formed, they cannot be the cause of pattern formation.

Fig. 4b compares four stress-strain curves in $\left[\begin{array}{lll}1 & 0 & 0\end{array}\right]$ multislip, with cross-slip included (bold
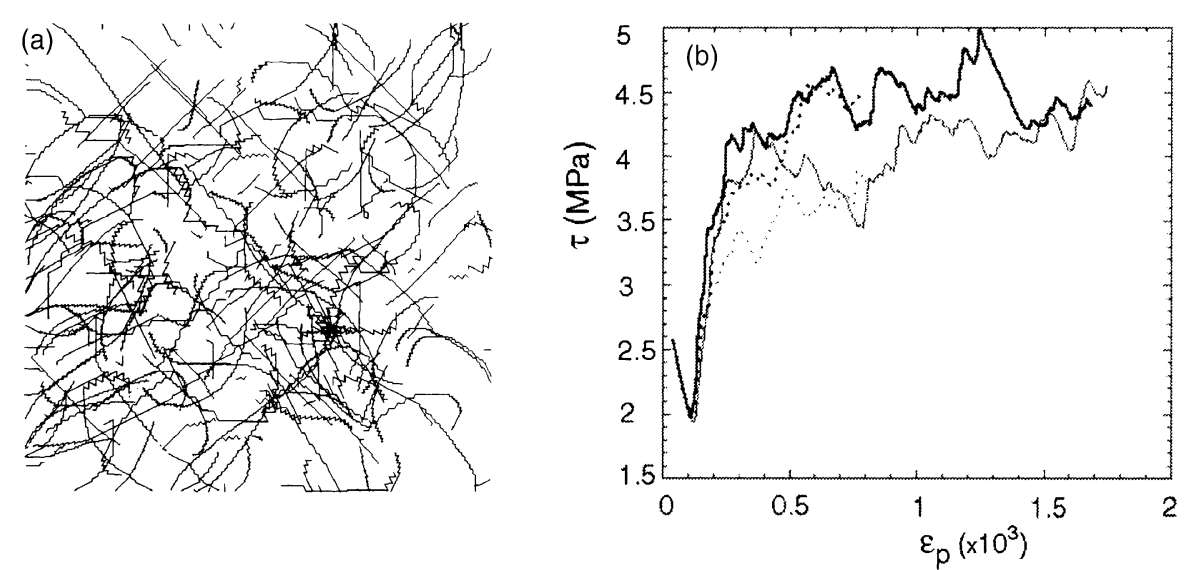

Fig. 4. (a) A simulated microstructure obtained in conditions similar to those of Fig. $2 b$ ([1 00 0 foil of thickness $4 \mu \mathrm{m}$ extracted from a simulation box of linear dimension $15 \mu \mathrm{m}$, Edge-Screw model). Elastic interactions between segments are computed only at distances larger than the mean dislocation spacing, $\rho^{-1 / 2} \approx 1 \mu \mathrm{m}$. Short-range interactions are truncated but junction processes are operative. A uniform dislocation distribution results. (b) Simulated resolved stress vs. plastic shear strain curves obtained in [1 00 ] multislip conditions using the Mixed model with cross-slip (bold curve), with cross-slip and only short-range interactions (bold dotted curve), without cross-slip (thin curve) or without cross-slip and with only short-range interactions (dotted thin curve). The initial stage corresponds to a relaxation of the initial configuration. 
curves) or not (thin curves) and with long-range interactions truncated (dotted curves) beyond a distance $\rho^{-1 / 2}=0.75 \mu \mathrm{m}$, or not truncated. Crossslip induces a small upwards shift in flow stress, which is attributed to the increased density of the forest via multiplication by double cross-slip and to the formation of jogs and debris. The longrange stresses do not significantly contribute to the flow stress. These conclusions are, of course, only valid in the small strain range considered here.

\section{Discussion and concluding remarks}

These results show that early stages of the organization of the microstructure can be investigated with the help of DD simulations. The dislocation arrangements obtained in this study share some properties of well-formed cells. Although it is not presently possible, by lack of computing power, to check how they evolve with increasing applied stress, they are associated with internal stress field patterns that behave as predicted by Mughrabi's composite model. Nevertheless, the flow stress of the simulated materials is governed by the forest mechanism, i.e., by shortrange and contact interactions. These patterns are stable within the small strain range investigated here. The dislocation processes that concur to the formation of these microstructures are, nevertheless, rather complex and have not been examined yet in detail.

As far as the modeling of dislocation pattern formation at low and medium temperatures is concerned, it seems essential to take into account not only the storage of dislocations by their interaction with the forest but also the cross-slip mechanism. Because cross-slip is stress-dependent, it is mostly sensitive to short-range dislocation interactions. Its effect is to decrease the mean internal stress by relaxing local peaks in its distribution, thus stabilizing the configurations of stored dislocations. All these features also characterize dynamic recovery, so that, in the absence of any obvious alternative, the present results support the view according to which the cross-slip mechanism is responsible for dynamic recovery. Cross-slip fa- vors non-planar glide and the present results also confirm that pattern formation is either delayed or suppressed in conditions of purely planar glide. Finally, long-range internal stresses, as well as local lattice rotations that are not included in these DD simulations, do not seem to critically influence the emergence of a cell structure.

There are virtually no transmission electron microscopy studies on single crystals deformed in multiple slip at small plastic strains, typically in the range of $0.5-1 \%$. It would certainly be desirable to undertake such a study in order to check the present conclusions regarding the bifurcation from an initially uniform dislocation structure to an organized one.

\section{References}

[1] Hirsch PB. In: Hirsch PB, editor. The physics of metals: II defects. Cambridge: Cambridge University Press; 1975. p. 193.

[2] Jackson PJ. Progr Mater Sci 1984;29:139.

[3] Kocks UF. In: Dislocations and properties of real materials. London: The Institute of Metals; 1985. p. 125.

[4] Kratochvíl J, Orlová A. Philos Mag 1990;A61:281.

[5] Zaiser M. Mater Sci Eng A 2001;309-310:304.

[6] Kubin LP, Canova G, Condat M, Devincre B, Pontikis V, Bréchet Y. Solid State Phenomena 1992;23-24:455.

[7] Devincre B, Kubin LP, Lemarchand C, Madec R. Mater Sci Eng A 2001;309-310:211.

[8] Madec R, Devincre B, Kubin LP. Multiscale Modeling of Materials, p. z1.8.1. In: Kubin L, Selinger R, Bassani J, Cho K, editors. MRS Symp Proc, vol. 653, Warrendale (PA): Materials Research Society, 2001.

[9] Madec R. Doctorate Thesis, Orsay University, 2001.

[10] Gómez-García D, Devincre B, Kubin LP. J Comput Aided Mater Design 1999;6:157.

[11] Bulatov V, Abraham F, Kubin LP, Devincre B, Yip S. Nature 1998;391:669.

[12] Shin CS, Fivel MC, Rodney D, Phillips R, Shenoy VB, Dupuy L. In: Forest S, van der Giessen E, Kubin L, editors. Scale transitions from atomistics to continuum plasticity. F-Les Ulis: EDP Sciences; 2001. p. 19.

[13] Bonneville J, Escaig B, Martin JL. Acta Metall 1988;36:1989.

[14] Vegge T, Rasmussen T, Leffers T, Pedersen OB, Jacobsen KW. Philos Mag Lett 2001;81:137.

[15] Devincre B. In: Kirchner HOK, Pontikis V, Kubin LP, editors. Computer simulation in materials science. Amsterdam: North Holland; 1996. p. 309, NATO ASI Series E308. 
[16] Bulatov VV, Rhee M, Cai W. Multiscale modeling of materials, p. z1.3.1. In: Kubin L, Selinger R, Bassani J, Cho K, editors. MRS Symp Proc, vol. 653, Warrendale (PA): Materials Research Society, 2001.

[17] Madec R, Devincre B, Kubin LP. Comput Mater Sci, 2002;23:219-224.
[18] Basinski SJ, Basinski ZS. In: Nabarro FRN, editor. Dislocations in solids, vol. 4. Amsterdam: North-Holland; 1979. p. 261.

[19] Devincre B, Kubin LP. Mater Sci Eng A 1997;234-236:8. [20] Mughrabi H. Acta Metall 1983;31:1367.

[21] Neuhaus R, Schwink C. Philos Mag A 1992;65:1463. 